\title{
Immunomodulation Therapy
}

National Cancer Institute

\section{Source}

National Cancer Institute. Immunomodulation Therapy. NCI Thesaurus. Code C116540.

Any treatment modality that exercises its effects by inducing, enhancing, or suppressing an immune response. 